\title{
Individuals' Decisions in the Presence of Multiple Goals
}

\author{
Benedict G. C. Dellaert ${ }^{1}$ (D) - Joffre Swait ${ }^{2}$ - Wiktor L. Vic Adamowicz ${ }^{3}$. \\ Theo A. Arentze ${ }^{4}$ Elizabeth E. Bruch ${ }^{5}$ - Elisabetta Cherchi ${ }^{6}$. Caspar Chorus ${ }^{7}$. \\ Bas Donkers $^{1} \cdot$ Fred M. Feinberg $^{8} \cdot$ A. A. J. Marley ${ }^{2,9} \cdot$ Linda Court Salisbury $^{10}$
}

Published online: 11 May 2017

(C) The Author(s) 2017. This article is an open access publication

\begin{abstract}
This paper develops new directions on how individuals' use of multiple goals can be incorporated in econometric models of individual decision-making. We start by outlining key components of multiple, simultaneous goal pursuit and multi-stage choice. Since different goals are often only partially compatible, such a multiple goal-based approach implies balancing goals, leading to a deliberate goal-level choice strategy on the part of the decision-maker. Accordingly, we introduce a conceptual framework to classify different aspects of individuals' decisions in the presence of multiple goals. Based on this framework, we propose a formalization of individual decision-making when pursuing multiple goals. We briefly review different previous streams on goal-based decision-making and how the proposed goal-driven conceptual framework relates to earlier research in discrete choice models. The framework is illustrated using examples from different domains, in particular marketing, environmental
\end{abstract}

Benedict G. C. Dellaert

dellaert@ese.eur.nl

Joffre Swait

Joffre.Swait@unisa.edu.au

Wiktor L. Vic Adamowicz

vic.adamowicz@ualberta.ca

1 Marketing Section, Department of Business Economics, Erasmus School of Economics, Erasmus University Rotterdam, PO Box 1738, 3000 DR Rotterdam, The Netherlands

2 Institute for Choice, University of South Australia, Adelaide, Australia

3 Department of Resource Economics and Environmental Sociology, University of Alberta, Edmonton, Canada economics, transportation, and sociology. Finally, we discuss identification and modeling needs for goal-based choice strategies and opportunities for further research.

Keywords Decision-making · Goal-based decisions · Multi-stage decisions · Choice modeling $\cdot$ Goal conflict

\section{Introduction}

Balancing multiple goals in life is a challenge that most of us are all too familiar with. On any given day, we strive variously to improve our health, develop our careers, connect more closely to loved ones at home, protect the environment, enjoy tasty food, and still find rest and peace within ourselves to reflect on who we are. This is no small task, and depending on external circumstances and the cognitive strategies that we employ, our achievement of
4 Urban Systems and Real Estate Group, Eindhoven University of Technology, Eindhoven, Netherlands

5 Department of Sociology, University of Michigan, Ann Arbor, USA

6 Transport Operations Research Group, Newcastle University, Newcastle upon Tyne, UK

7 Transport and Logistics group, Delft University of Technology, Delft, Netherlands

8 Ross School of Business and Department of Statistics, University of Michigan, Ann Arbor, USA

9 Department of Psychology, University of Victoria, Victoria, Canada

10 Carroll School of Management, Boston College, Chestnut Hill, USA 
these different goals likely varies over time. An urgent deadline at work will force us to prioritize career-related goals, while imposing a strict self-control regime will allow us to exercise every day to support our health-related goals. The different goals we select and how we prioritize them affect our behavior and the choices that we make [4, $13,17,28]$. Yet only recently has research begun to address the question of how goals can be directly incorporated in econometric models of individual decisionmaking to test theories about goals and improve our understanding and prediction of individuals' choices (e.g., $[35,53])^{1}$

We illustrate the challenge of combining goal-based theory and modeling individuals' choices of alternatives through an example. Assume a person is choosing a dessert to buy for a dinner at home and uses two personal goals in the selection: avoid gaining weight and indulge in something tasty. The person might decide to set a threshold on each goal and only include desserts that exceed both thresholds (e.g., a tasty and healthy tropical fruit dessert), or the person may choose-for the one special evening only - to focus exclusively on the goal of tastiness (e.g., a rich chocolate brownie mousse). Note that at the goal activation level, the person need not yet assign a unitary value to any dessert alternative, but decides on the goal strategy level first; that is, from the perspective of goal pursuit, a strategy can be defined without consideration of specific means (or options) of achieving them. Now change the framework from attainment of the two goals to the more traditional choice modeling view of all possible desserts having a utility value, which is a function of each dessert's attributes. Then the model for the selection would be to choose the dessert with the highest overall utility value (i.e., comparing tropical fruit to brownie mousse) without taking into account the higherlevel goal trade-offs. By ignoring the goal-level decision strategy, researchers, managers, and policy makers would develop an inaccurate understanding of how the individual's decision is made, what might change the individual's decision (in terms of context, communications, and alternative designs), and hence how healthier outcomes for the individual could best be promoted.

The aim of this paper is to further developments in this direction by integrating research on individuals' simultaneous use of multiple goals with current advances in choice modeling. The theoretical embedding of goalbased strategies in models of choice is important for a number of reasons. ${ }^{2}$ First, choice models that account

\footnotetext{
${ }^{1}$ At this stage, we deal with the term "goal" in an informal and colloquial fashion. We provide a more rigorous definition of the construct in the next section.

${ }^{2}$ We thank an anonymous reviewer for constructive suggestions that helped us in more clearly highlighting these three points.
}

for individuals' goals may lead to better predictions of consumer behavior. If different goals are (de)activated by individuals, they may employ different decision strategies, different environmental cues may be important to them, they may search different information sources, and ultimately, they may select different alternatives. Thus, behavioral differences between individuals, or within an individual at different points in time or contexts, may depend on the goal-based strategies that they follow. Second, these models may lead to new insights and explanations of behavior that cannot be accomplished by simpler models (of either goal activation or choice alone). For example, goals are also used by individuals to determine the allocation of scarce (cognitive) decision-making resources they have to arrive at better choices [60]. In particular, the difficulty of making a decision due to personal restrictions (say, cognitive or budgetary limits), exogenous constraints (e.g., time and monetary limits), and contextual complexities (e.g., large numbers of alternatives or many aspects) can be addressed through the introduction of multi-layered decision strategies aligned with goal hierarchies and/or goal priorities. Third, the new models may lead to new recommendations to managers or policy makers that cannot be uncovered by a simpler model. In particular, by better understanding differences in goal activation, managers and policy makers who wish to support individuals in making better decisions (e.g., by living healthier lives, planning their finances better, or selecting between education options) may be able to develop more effective policies and provide better assistance. When individuals look for guidance in achieving better choice outcomes, a purely predictive model at the level of their current alternative choices is of limited use in terms of guiding and improving decisions. In contrast, a goal-oriented model is needed to also understand what individuals are hoping to achieve and what goal-based decision strategy they would like to employ to make their decisions.

Thus, based on workshop deliberations, we believe that the explicit extension of econometric choice models to include simultaneous multiple goals will result in three types of benefits compared to extant microeconomic and psychology theories: (1) consumer decision outcomes will be more accurately predicted; (2) enhanced understanding about decision processes; and (3) improved insights about consumers, hence novel policy recommendations. While at this early stage goal-based choice models are in their infancy, and these claims are yet to be firmly established, early empirical work is encouraging that the proposed goal-based extensions to choice models will deliver on them.

Turning our attention to the remainder of the paper, we next outline the key components of multiple, simultaneous goal pursuit and multi-stage choice. Since different goals are often 
only partially compatible, such a multiple goal-based approach implies balancing goals, leading to a deliberate goallevel choice strategy on the part of the decision-maker. Therefore, we introduce a conceptual framework to begin to classify different aspects of individuals' decisions in the presence of multiple goals, with an eye towards tying contextual and other covariates into predicting goal (de)activation as an antecedent to eventual choice. This framework allows us to also propose a useful formalization of individual decisionmaking when pursuing multiple goals. Next, we briefly review different previous streams on goal-based decision-making. In addition, we review how the proposed goal-driven conceptual framework relates to earlier research in discrete choice models. We further illustrate the framework using examples from different domains, such as marketing, environmental economics, transportation, and sociology. Finally, we discuss identification and modeling needs that arise from the question of whether incorporating goal-level choice strategies can indeed improve the insights and predictive performance of models of individual choice.

\section{Setting the Groundwork for a Goal-Driven Choice Model}

In this section, we present and describe the goal-based model of choice that the workshop participants evolved towards during the course of the Choice Symposium. We do this up front because this model frames the remainder of the paper and clarifies that we are ultimately working towards the formulation of choice models that are not simply goal-sensitive, but are fundamentally goal-driven. ${ }^{3}$

The psychology literature on goals is vast and long-standing, as evidenced by the now classic review of Austin and Vancouver [4]; it has only increased since then (see, e.g., [60]). The former authors state: “. $\ldots$ the sheer magnitude of this body of research is associated with a certain danger. Heterogeneous perspectives can generate a large body of facts, an excess of vocabulary, and numerous micro theories ..." (see p. 338 in [4]). Thus, before undertaking the task of conceptualizing a choice framework based on goal pursuit, it behooves us to spend some effort defining what we mean by "goals." It seems most useful to start by recognizing that many types of constructs of interest to psychologists and social scientists might be expressed as, or reflected in, expressions of goals. For example, needs (e.g., hunger, thirst, entertainment) or values (e.g., statements about high-level abstract personal characteristics that we would like to possess) can be expressed as goals, by including the construct in a statement embodying pursuit of a desired end state. Fundamentally,

\footnotetext{
$\overline{3}$ Van Osselaer and Janiszewski [58] present a framework for goal-based choice that is motivated by ideas and concerns similar to our own.
}

goals are directive in nature, in that their pursuit will lead to desirable end states. More effectively, however, a taxonomy that recognizes that there exist functional goals whose attainment is directly linked to product or service characteristics seems most useful for our purposes. More abstract (i.e., non-functional) goals reflect the existence of a goal hierarchy that will require a cascading of sub-goals that ultimately leads to a set of functional goals as they operationalize the abstraction into ever more specific pursuits. Another distinction with a practical meaning for our purposes is that goals can be approach or avoidance goals [4, Table 2], indicating that their "valence" has a behavioral impact. Finally, we wish to point out that decision-makers can pursue functional goals (those they are trying to accomplish through the decision being made) as well as process goals (those they are attempting to pursue that have to do with how the decision is being made). In any particular modeling context, the focal goals may come from multiple categories depending upon the problem features that are of greatest research and substantive interest.

We begin by introducing the notion that a goal-driven choice requires operating in two separate but related spaces (see Fig. 1): (1) attribute space (with dimensions $x_{1}, x_{2}, \ldots$ ), where alternatives $\left(z_{1}, z_{2}, \ldots\right)$ exist, and (2) goal space $\left(G_{1}, G_{2}\right.$, ...), with dimensions determined by the decision-maker. Note that the standard micro-economics description of decisionmaking restricts its attention to attribute space and overlays a value (or utility) function defined on attributes; the highest value option is (usually) presumed selected. This depiction of the choice process also comports the concept of attributebased constraints, which leads to the creation of a choice set $\Upsilon_{X}$ of feasible alternatives (the $X$ subscript refers to the attribute space, as defined above). The generalization of the choice process from the point of view of goals is depicted in Fig. 1. Specifically, choice arises from a goal set $\Gamma_{\mathrm{A}} \subseteq \Gamma$ ("A" for activated and with $\Gamma$ a (currently vaguely defined) notional super set of goals). The activation of goals results in a definition of the metrics of success for the choice problem at the goal level.

In Fig. 1 , goals $G_{1}, G_{3}$, and $G_{4}$ are activated by the decision-maker, whereas $G_{2}$ is not (for example, this could be due to a lack of relevance to the decision or inability to attain it with the options presented/available for choice). The doubleheaded block arrows between the attribute space and $\Gamma_{\mathrm{A}}$ are there for the purpose of reminding us that the (de)activation of goals will be influenced by the attribute space via the alternatives, which in turn may be influenced by the imposition of attribute-driven constraints reflecting one or more goals (e.g., a price limit).

The core of the proposed framework is depicted in the goalspace diagram. We highlight three important points about this framework. First, and perhaps foremost, the activation and pursuit of multiple goals is directly represented in this space, without appeal to some kind of value function. Second, 


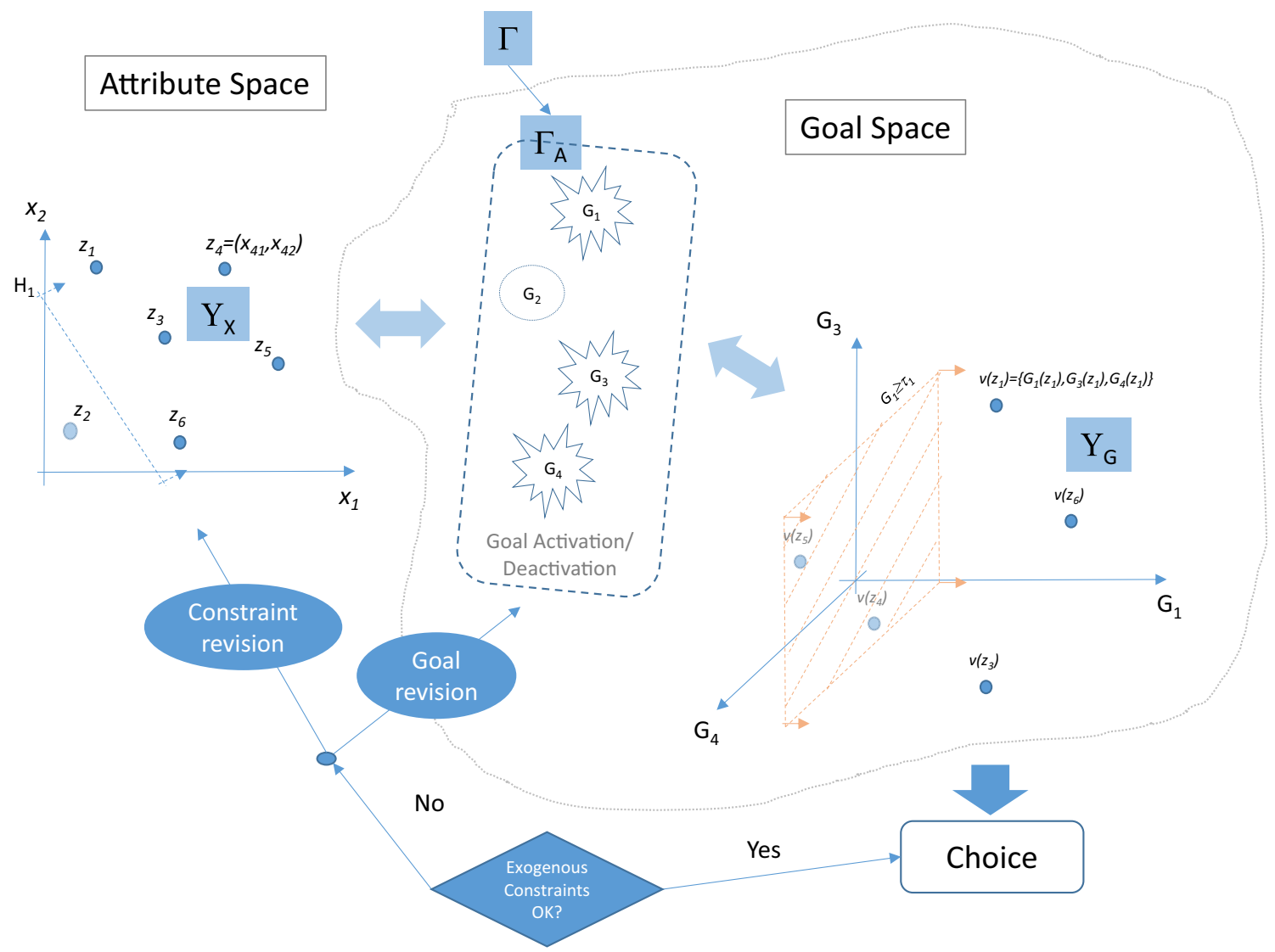

Fig. 1 Framework relating multiple-goal pursuit to choice

decision-makers can consider goals met or not for different reasons: one goal may require that a threshold level be met, whereas another may need to be pursued to best end (i.e., optimized). More generally, goals can be constraints and/or objectives. For example, the figure makes the point that goal $G_{1} \geq \tau_{1}$, which leads to the further elimination of alternatives $z_{4}$ and $z_{5}$ (to remind, $z_{2}$ was eliminated due to constraint $H_{1}$ in attribute space), i.e., the choice set of feasible alternatives in attribute space, $\Upsilon_{X}$, can be further refined in goal space to set $\Upsilon_{G}$. This need not happen sequentially, and each of these sets can have unique elements. Third, decision-makers' choice of establishing the role of goals, singly and in interaction, is what we suggest be termed the goal choice strategy. That is, to make a product choice, the decision-maker must make two fundamental determinations with respect to goal space: (1) What are its dimensions (e.g., activate $G_{1}, G_{3}, G_{4}$, but not $G_{2}$ ) and (2) What constitutes the attainment of each goal (e.g., maximize $G_{1}$, set a target on $G_{3}$, and keep $G_{4}$ above a threshold). These decisions are of prime importance because they reflect the presumption that the decision-maker's choice is ultimately determined in goal space, not attribute space, as commonly represented.

The mechanics of determining the level of attainment of $G_{k}(z)$ by an alternative $z$ is what we term the goal evaluation strategy. The goal evaluation strategy may be a function of some or all of the attributes that affect the achievement of alternatives on that goal, and the goal-specific strategy may differ across goals. For example, the tastiness of a dessert may be determined by a combination of its sugar and fat contents, its flavoring, etc.; its healthiness, for a gluten-intolerant individual, may be determined simply by whether or not it contains wheat.

Thus, the goal evaluation strategy does not affect the choice directly but determines how well alternatives perform on each of the different goals. The goal choice strategy then drives the choice by combining the impact of the different goal evaluations on choice. A noteworthy point to make here is that the goal choice strategy is not necessarily a function of anything involving the lower-level outcomes (not the options, or their attributes, or the levels of those attributes, or how one uses those to create a single evaluative scale, such as "utility"). That is, if one decides to have a healthy dessert, one does not necessarily first ask about which healthy desserts are available.

Note that in this formulation, we have employed approach goals or goals that decision-makers select to achieve or satisfy. The model could also be formulated with avoidance goals, as mentioned above, with the corresponding change in algebra. However, one may also consider behavioral aspects of approach versus avoidance goals. Heterogeneity may include 
individuals who choose to achieve certain outcomes (healthy body weights), or avoid outcomes (obesity), or who choose to achieve personal safety or avoid risky situations. The choice process outcomes may differ for those who formulate these goals in the approach mode or avoidance mode, perhaps somewhat akin to the outcomes arising from framing choices as gains or losses.

To illustrate these concepts, suppose a decision-maker might decide on the strategy of compensatory pursuit of goals $G_{3}$ and $G_{4}$ (refer to Fig. 1), using weights that sum to unity, while requiring that $G_{1} \geq \tau_{1}$ and $H_{1} \geq d$, where $\tau_{1}$ and $d$ are two known thresholds. The goal choice strategy for two goals $G_{3}$ and $G_{4}$ then is the following:

Choose $i \in M$ such that

$$
\begin{array}{cl}
\max _{i \in M} \Psi_{M}=\sum_{i \in M} \delta\left(w_{3} G_{3}\left(X_{i}\right)+w_{4} G_{4}\left(X_{i}\right)\right) \\
\text { s.t. } \\
0 \leq \delta_{i} \leq 1 \forall i \in Y_{X} \cap Y_{G} \\
\delta_{i}=0 \forall i \notin Y_{X} \cap Y_{G} \\
\sum_{i \in M} \delta_{i}=1 \\
\mathrm{Y}_{X}=\left\{i \in M \mid H_{1}\left(X_{i}\right) \geq d\right\} \\
\mathrm{Y}_{G}=\left\{i \in M \mid G_{1}\left(X_{i}\right) \geq \tau_{1}\right\}
\end{array}
$$

In the goal-based decision problem (1), the objective function is specified as compensatory; it could just as easily be defined as some non-compensatory function. For example, if the strategy were changed to choose the option that disjunctively optimizes either $G_{3}$ or $G_{4}$, we simply change the objective function in program (1) to

$$
\max _{i \in M} \Psi_{M}=\sum_{i \in M} \delta_{i} \max \left(G_{3}\left(X_{i}\right), G_{4}\left(X_{i}\right)\right)
$$

In our desire to work towards workable operational choice models of goal-driven behaviors, formalisms of the type represented by (1) make it possible to envision model specifications that directly reflect goal constructs. As we see above, mathematical program (1) is capable of (a) incorporating goals both as objectives $\left(\Psi_{M}\right)$ and constraints $\left(Y_{G}\right)$, (b) handling multiple-goal pursuit (exemplified here with goals $G_{3}$ and $G_{4}$ ), and (c) including attribute-space constraints $\left(Y_{X}\right)$. A decision may also be subject to what one might term "side" constraints, which are neither attribute- nor goal-spaced based, such as a budget constraint. This extension requires the introduction of constraints to (1), such as

$\sum_{i \in M} \delta_{i} p_{i} \leq I$

where $p_{i}$ is the (exogenous) price of good $i$ and $I$ is the decision-maker's income.

Figure 1 shows one representation that extends our basic goal-driven decision framework to handle such constraints: if the preferred option in goal space meets these requirements, the choice made in goal space is final; if not, it is necessary to revise goals (e.g., activate or deactivate some goal, change the relative importance of one goal relative to others), change goal choice strategy or goal evaluation strategy, introduce or relax constraints in either attribute or goal space, etc.

An important point about Fig. 1 merits repetition: this framework is meant to imply that choice is determined in goal space, not attribute space. The framing of the decision problem is made in terms of the goals and how they are to be used to make a choice - i.e., determination of the goal choice strategy; secondarily, how individual goals are "calculated" is established-i.e., determination of the goal evaluation strategy. Attribute space contributes essential information about constraints and other relevant information, but it is not where choice is determined.

\section{A Brief Review of Goal-Based Decision Models}

\subsection{Empirical Models}

There is burgeoning empirical and theoretical research on goal-based choice at the intersection of cognition and brain sciences, neuroscience, and neuroeconomics, both for individuals making decisions in isolation and in social situations [20].

A recent example of research into functional goals is Larrick [30] who presents a framework for decisions in social contexts similar to that considered in the present article. He introduces "Objectives to achieve with the decision" (whereas we conceptualize goals as desired levels of attainment of objectives) and presents attributes as "indicators/means of achieving objectives." Another example is Orehek and Forest [43] who include relationship partners as means to goals and suggest that satisfying relationships are achieved when partners experience mutually perceived instrumentality - that is, each partner feels instrumental to his or her partner's important goals and simultaneously perceives the partner as instrumental to their own important goals. This perspective guides their predictions about how people cope with relationship loss (e.g., due to death of a partner) - individuals with social networks that can substitute for a lost partner should find partner loss less devastating. Other related recent work by Etkin [14] studies variety goals in committed relationships; for example, when planning a weekend together, one member of a couple could choose more varied activities (e.g., going out to dinner, to a movie, and to a concert) or less varied activities (e.g., going to three different movies). Etkin's experimental work shows that when consumers perceive more (respectively less) future time ahead in a (committed) relationship, they prefer more (respectively less) variety in joint consumption; the studies also reveal different choices for solo versus joint consumption. As the author notes, these results have 
implications for recommendation systems. For example, suppose a couples' most recent vacation was a Caribbean cruise, then a good recommendation for a younger relationship would be a very different trip (e.g., a wine tour) and, for an older relationship, a similar trip (a cruise to a different place). This prediction seems to run counter to popular expressions such as "you only live once" and the notion of a "bucket list" and suggests the need for further studies that control for how much variety a couple, and the individuals in the couple, have achieved to date.

A person's values are the principles or standards of their behavior and involve their judgment of what is important in life; thus, they might be considered to be determinants of their objectives and/or goals. Kasser [24] assumes that the value of materialism "reflects the extent to which an individual believes that it is important to acquire money and possessions, as well as to strive for the related aims of an appealing image and high status/popularity, both of which are frequently expressed via money and possessions"; he presents research on participants' ratings of the importance of a variety of goals, with materialism assessed by calculating the importance of extrinsic goals for financial success ("I will be financially successful"), image ("My image will be one others find appealing"), and popularity ("I will be admired by many people") relative to other goals.

When we look at process goals, a habit leads a person to repeat the same behavior in a recurring context. Adamowicz and Swait [1] characterize habits as decision strategies that minimize cognitive effort, and Wood and Runger [61] characterize them in terms of their cognitive, motivational, and neurobiological properties; the latter review summarizes computational models of habit and deliberate goal pursuit, with habit the learned efficient default mode of response. The main classes of models presented are based on (1) artificial neural networks with two interlinked habit and goal networks [10]; (2) a cognitive architecture within the adaptive control of thoughtrational (ACT-R) framework where behavioral control shifts from an internal, declarative task representation (goal based) to one based on environment cues (habit based) [54]; and (3) cognitive neuroscience models of reinforcement learning (RL) where goal-directed learning involves mental simulation and planning (model-based learning) and habit formation involves trial-and-error learning to estimate and store long-run values that are available in different states or contexts (model-free learning) [18].

Cognitive neuroscience RL models are driven by, and in turn drive, neuroscientific research using functional neuroimaging with human subjects, with the latter providing evidence on neural systems that integrate habit and goal-directed action (see Wood and Runger's section Neurobiology of Habits). O'Reilly et al. [40] present a computational framework in which goal-driven behavior is in place from the start, not arising solely in reaction to the environment as it is typically conceived in RL models. Their main hypothesis is that there are two qualitatively different states of mental life: goal selection where a decision-maker carefully considers the cost and benefits of possible goals and goal engaged where a decisionmaker has decided to try to achieve one or more goals. They assume the neural system is motivated to have at least one goal engaged, and once a goal is engaged, it dominates the evaluation of options and resists disengagement. The framework is implemented in a biologically constrained computational algorithm, with the constraints motivated by known features of relevant brain areas and mechanisms, and can potentially explain a wide range of clinical disorders. There is extensive related work in Neuroeconomics, mainly studying brainbased representation and learning of values; in particular, see Glimcher and Fehr [20] and the review by Konovalov and Krajbich [27].

\subsection{Normative Models}

The analysis of decision-making has received equal attention from the perspective of prescriptive or normative models. From the prescriptive perspective, the aim is to develop models that can support decision-making, rather than to describe or predict it. In prescriptive approaches, the notion of goals of the decision-maker plays a central role, as without knowledge of the decision-maker's goals it would be impossible to identify what the best decision is in any given decision problem. Being able to generate a list of the objectives to be achieved by the decision of concern is an axiomatic assumption of decision analysis that is part of the foundation of the field as put forward by Von Neumann and Morgenstern [59] and Savage [47]. As Keeney [25] puts it "if the set of objectives has not been generated then there is no basis to even think about which alternatives may be better than which others and why this is the case." Thus, where one could possibly disregard goals in descriptive approaches, they constitute the cornerstone of prescriptive frameworks.

To discuss how the notion of goals has been incorporated in prescriptive decision models, it is helpful to make a distinction between two phases in decision-making processes, namely (1) structuring the decision problem and (2) evaluating the decision alternatives for making a choice. Decision support methods generally intend to support both phases. Knowing one's goals or objectives is essential to structuring a decision problem, as goals provide the basis for both identifying decision alternatives (creating a choice set) and specifying preference functions for evaluating the alternatives. Failure to identify underlying objectives is a well-known pitfall in decisionmaking [7]. Developing a goal hierarchy is an example of a method that is advocated to avoid overlooking essential evaluation criteria. In this method, the user creates a goal tree by iteratively deriving sub-goals from main goals (of the 
organization) until they are operationalized as criteria on which alternatives can be evaluated [46].

The adoption of goal-based approaches in the choice phase is less evident in multi-criteria decision analysis. A variety of approaches have been developed for supporting evaluation and making a decision. Different standpoints are taken in approaches with respect to the question whether value can be represented through a closed-form preference function. Multiattribute Utility Theory (MAUT) and Analytical Hierarchy (AHP) are two mainstream approaches where value is expressed through a linear additive (closed-form) function of attributes/criteria. MAUT models assume a utility function similar to the linear-in-parameters utility functions assumed in (descriptive) discrete choice models. AHP also uses the idea of a closed-form evaluation function. However, the approach to estimating relative values of criteria as well as scores of alternatives differs (i.e., a geometric mean or eigenvalue method is used). On the other hand, outranking methods, a third important category of multi-criteria decision analysis methods, do not assume a closed-form evaluation function. Outranking methods emphasize that a complete ranking of alternatives on the given decision criteria may not be feasible. Their purpose is to perform an initial screening and produce a short list that is presented to the decision-maker for a thorough trade-off [41].

The closed-form evaluation functions as used in MAUT, AHP, and other approaches generally assume a compensatory structure. Non-compensatory decision rules, on the other hand, have also received attention especially in case of decision problems where the number of decision alternatives is large or the solution space is not even continuous. In goal programming, the decision problem is structured by means of a goal achievement function where a target value is defined for each goal of the decision at hand and the best solution is defined as the solution that minimizes the total deviation from targets across the goals. Although many forms of noncompensatory decision rules are possible, the lexicographic rule and the minimax rule are the most commonly used noncompensatory rules in goal programming [45]. In the case of a lexicographic rule, no trade-offs are made between goals that are placed in different priority levels, whereas if a minimax rule is used, the maximum deviation across goals is minimized (a solution is as good as the performance on the goal on which it performs poorest).

To conclude, what evidence do we find for goal thinking in prescriptive approaches to decision-making? As the above short review suggests, goal thinking is evident in the decision problem structuring phase: knowledge of decision-making goals is considered essential for defining preference or goalattainment functions to evaluate alternatives. In MAUT, AHP, and other approaches that assume linear additive evaluation functions, goals are less central to the approaches but implicitly represented in functions that define performance of alternatives typically in attribute space. Outranking methods are more conservative and assume that some trade-offs may be too complex to be made in such a fashion and leave those to the decision-maker. Furthermore, in goal-programming approaches, goal achievement functions assuming more complex trade-offs in goal space are relatively standard.

\section{The Proposed Goal-Driven Model of Choice Relative to Other Discrete Choice Models}

In this section, we briefly review the relationship between our proposed conceptual framework and traditional discrete choice models and in particular how the latter have been expanded over the years to incorporate various behavioral effects. We focus on the systematic component of the discrete choice models. While much research has also addressed random error components in models of choice to capture heterogeneity in taste (e.g., random coefficient or mixed logit models, see [37]) or heteroscedasticity between decisions or individuals (e.g., the generalized multinomial logit model [16]), in this overview, we are mainly concerned with the structure of the systematic components in the model that capture variations in decision processes or decision weights.

The traditional discrete choice model connects alternatives' attributes and their levels to the underlying latent utility that consumers derive from these levels ([36] - see Fig. 2). Alternatives can differ in the attribute levels that they offer (e.g., consumer products, transport modes, jobs, potential romantic partners). Individuals evaluate the attractiveness of these levels and integrate these evaluations into an overall utility that they attach to each alternative. Individuals' choices depend on the overall utility per alternative, and they choose the alternative with the highest utility subject to their financial budget and their time constraints.

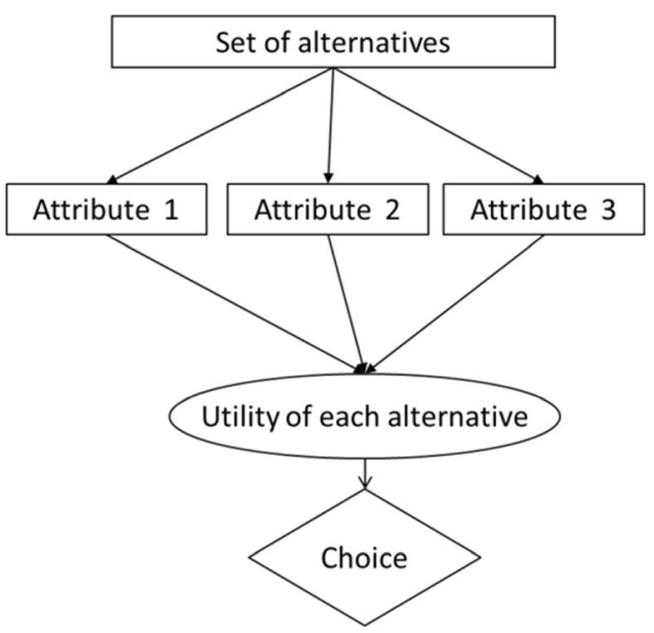

Fig. 2 Structure of traditional discrete choice models 
Based on insights into behavioral effects in individual decision-making, such as the use of heuristics or evaluation biases, these traditional discrete choice models have been expanded to also allow for the use of different decision rules. For example, flexible models have been proposed to capture whether individuals use conjunctive or disjunctive decision rules [19,22]; shifts in decision rules based on the complexity of the decision task $[12,51]$; the use of cutoffs in decisions [50]; and the impact of regret on decisions [9]. These models have in common that they augment the traditional discrete choice model with an additional modeling layer that captures variations in how different attribute levels are integrated in a heuristic decision rule that results in an individuals' choice of an alternative (see Fig. 3).

Recent work has taken a different angle on extending discrete choice models: instead of focusing on decision heuristics, it has addressed the question what higher-order benefits consumers look for when making decisions. This research builds on early work in, e.g., marketing, which highlighted that consumer needs may vary across situations (e.g., [39]) and that the evaluation of attribute levels may depend on the degree to which they can help fulfill underlying consumer needs (e.g., $[11,21,56])$. Researchers have proposed different models that can capture this intermediate layer in the utility formation process. Hybrid choice models have been proposed that flexibly incorporate such intermediate evaluation steps [6]. These evaluations have been treated as latent (benefit) variables by some researchers [26, 44], while other researchers have explicitly elicited attribute-benefit connections from individuals [2]. Thus, in these models, the attributes of each alternative drive how the alternative is evaluated on each of a set of different benefits that consumers look for and the utility that is subsequently attached to the different benefits determines the consumer choice. Figure 4 summarizes the structure of this second stream of choice model extensions.

In this article, we propose a different approach to the development and motivation of discrete choice models that is conceptually related to benefit-based discrete choice models

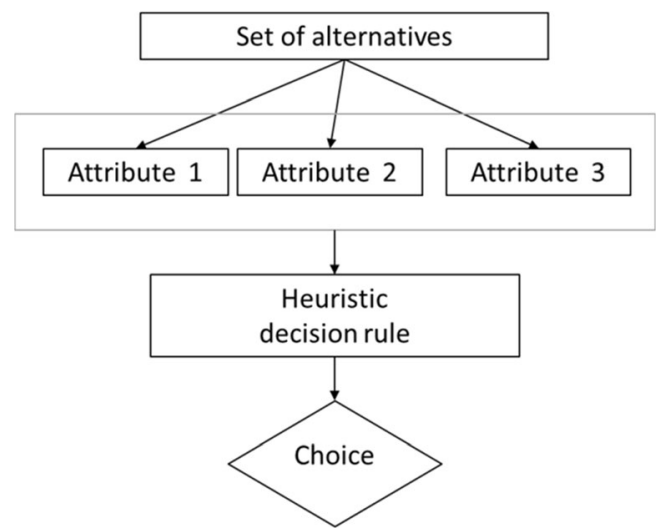

Fig. 3 Structure of behavioral discrete choice models that allow for decision heuristics

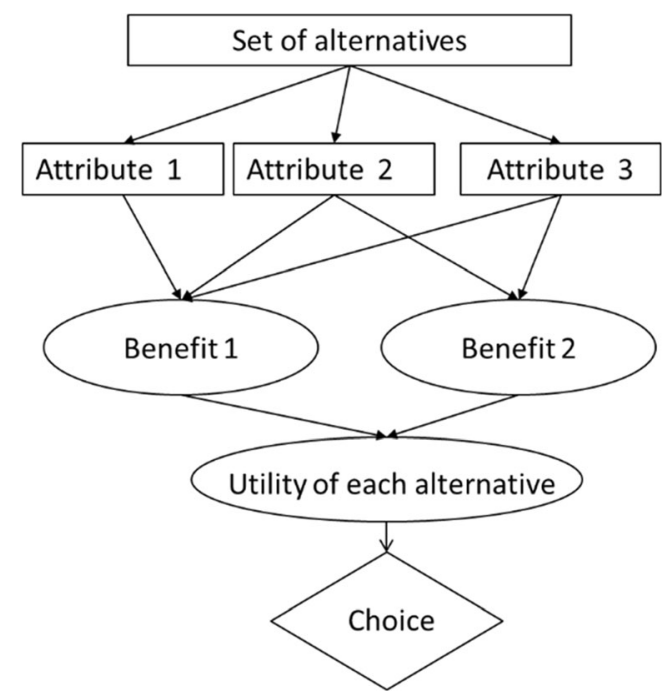

Fig. 4 Structure of benefit-based discrete choice models

but is also fundamentally different in that it explicitly acknowledges that individuals may also form decision strategies at the level of the goals they pursue. More precisely, we view a goal as conceptually similar to an individual's desire to achieve a certain benefit (e.g., an individual's goal of eating healthily aligns with looking for the benefit "healthy" in food; an individual's goal of saving effort aligns with looking for the benefit "convenience" in transportation; etc.). However, we propose that achieving goals is not a neutral process that translates directly to utility (such as in the benefit-based models), but that much as in the case of heuristics and attribute-level evaluations, individuals may choose to follow a certain goal strategy to come to a choice. For example, they may focus on one goal at the expense of all others, or they may balance different goals when making a decision. Thus, in evaluating alternatives, individuals follow a two-step approach, for which the order of cognitive processing may vary. Step 1 is the goal evaluation strategy in which alternatives are evaluated in terms of how well they perform in achieving a certain goal (e.g., are Brussel sprouts healthy? are they tasty?). Step 2 is the goal choice strategy, in which the individual determines how to integrate the fulfillment of different goals into the selection of an alternative (e.g., focus exclusively on health outcomes of food or balance healthiness against tastiness). This proposed structure is graphically summarized in Fig. 5 and was formalized in the proposed conceptual model at the start of this paper.

\section{Applications in Different Fields}

\subsection{Marketing}

As an example from marketing on the impact of goal choice strategies, consider the case of a consumer's choice of a new home. Multiple (often competing) goals are likely activated 


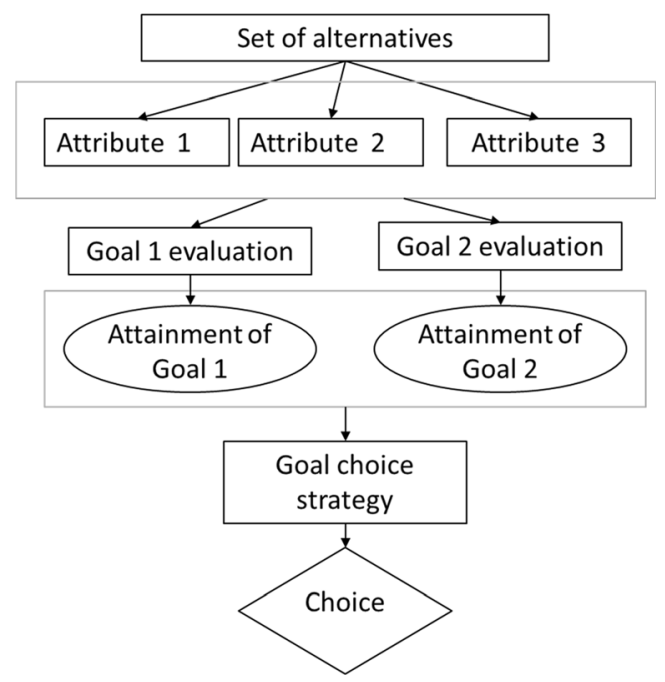

Fig. 5 Structure of goal-based discrete choice models

when someone is choosing a new home, and activated goals will constrain the feasible attribute space. For example, a desire to achieve or maintain financial security creates a home price ceiling (i.e., rent, mortgage), while other competing goals such as achieving social status or having welleducated and accomplished children translate into a price minimum threshold and neighborhood location constraints (e.g., acceptable school districts). A work-family balance goal may further constrain neighborhood location (i.e., commute time) as well as property size and condition (i.e., required maintenance and repair time). Household budget constraints limit the feasible attribute space and goal space, as illustrated in Fig. 1, especially for low- and middle-income households, and goal importance/activation is likely to be updated as the choice process progresses. Heterogeneity in the relative importance, or activation, of each goal will manifest in different decision strategies across a cross section of consumers. Similarly, we expect to observe heterogeneity within households for repeated home choices over time as the relative importance of each goal evolves over the lifetime of the decision-maker.

\subsection{Environmental Public Policy}

Two examples of areas in environmental economics that employ models of choice and in which goal choice strategies may be impactful are recreation demand models (intended to capture the behavior associated with recreation site choice and measure values arising from changes in environmental attributes) and models of passive use value (intended to capture monetary values for changes in public goods or publically provided goods). In both of these cases, multiple goals may play a significant role in decision-making. In the recreation context, goals may include fitness, socializing, desire for solitude, developing skills (e.g., rock climbing or birdwatching), passing on traditions to children (e.g., hunting or fishing skills), or expanding experiences (e.g., life lists for birdwatchers). These goals may compete with each other and may differ across social contexts (family trips versus trips with colleagues). Morey and Thiene [38] examine such recreational choices in the context of "life constraints," but this approach can be re-considered in a goal-based framework. In the elicitation of passive use values, which is typically framed as a referendum between policy options, there is a long history of discussion of the competing goals of being a "citizen" (altruistic, socially minded) versus a "consumer" (rational self-interested individual). These different goals have been examined empirically [5], and conceptual models have been developed to reflect these goals and the factors that affect the balance between them. Levitt and List's [31, 32] model that outlines conflict between moral/ethical goals and personal wealth goals has been used as a framework to understand choices in passive use value (as well as other) contexts.

\subsection{Transport}

The role of goals in transport choice has been studied in the context of sustainable transportation mode choice. Individuals who cherish feelings of power and pleasure (power and hedonic goals) put a high relevance on owning their own transport mode, which positively affects their choice of using their private car for trips [44]. Individuals who are interested in pleasure and sensuous gratification for oneself right now (hedonic and egoistic goals) are more likely to use the automobile instead of adopting pro-environmental behaviors [49].

Competing goals may also be activated in particular situations. For example, in choosing the type of car to buy, a goal of behaving in an environmentally friendly manner which would lead, say, to the choice of an electric vehicle can compete with a goal of achieving social status, which might lead towards the choice of a luxury car. Since luxury electric vehicles are extremely expensive, achieving both goals might be limited by the individual's budget constraint. Individuals' final choices will depend then on their goal choice strategy and how each alternative is evaluated on the different goals (i.e., goal evaluation strategy).

\subsection{Sociology}

As an example in the domain of sociology, multiple, potentially competing goals are likely activated when searching for a romantic relationship. For example, a woman seeking a marriage partner might restrict her search to men who are financially secure to increase her own financial security as well [42]. But the pool of financially secure men tends to be older than the general population; pursuit of this goal would increase her likelihood of ending up with someone much older and previously married, which could conflict with the goal of having a conventional (non-blended) family arrangement. 
Moreover, a woman in the dating market may have multiple goals that span very different time horizons, constraining the attribute space. For example, she might eventually wish to be married, but in the short term wants to have fun and explore her options. In selecting a goal choice strategy, she must decide how much effort to allocate to achieving these two goals, and this in turn may imply very different subsets of the attribute space that she will consider. Different mate seekers may allocate different levels of effort to these two goals, resulting in heterogeneous goal choice strategies.

It is interesting to note that, while goals constrain attribute space, attribute space may also constrain the emphasis put on certain goals and hence the goal choice strategy. For example, the local dating pool is a major constraint that limits feasible attribute space, as it determines who is available to mate seekers. What goals are activated may also depend on some overall evaluation of the set of available options-i.e., whether the total available set is deemed overall desirable or undesirable (e.g., [34]). When desirable options are thin on the ground, a mate seeker may abandon the goal of finding a long-term partner and instead focus on pursuing more short-term encounters. Thus, in the presence of multiple goals, if one goal constrains attribute space too much, the decision-maker may shift her focus to goals where she is more likely to succeed.

\subsection{Specific Examples of Implementation}

To focus the applications further, we present two recent examples of the application of such steps towards goal-based research. They are Swait et al. ([52] based on Li [33]) and Souza [48]. The former developed an econometric model for latent (functional) goal identification using traditional stated preference (SP) data. Apropos the issue of validation, Swait et al. [52] employ one discrete choice experiment (DCE) concerning hypothetical digital camera profiles to estimate model parameters, then use a second DCE with real digital cameras on an independent sample from the same population to conduct out-of-sample forecasts. They find that the out-ofsample forecasting of the goal-based model is markedly superior to a standard latent preference class model. Similarly, Souza [48] investigates the screening role of (latent) goals in choice set formation. Following model estimation, he uses content analysis of user-provided motivations for screening to validate econometric inferences. Both references illustrate the usefulness and increased precision of interpretation arising from a goal-based modeling approach, reinforcing that researchers should plan robust validation exercises as part of model development and testing. A further application in the environmental economics area is Thiene et al. [55] who examine the role of goals in choice set formation. They include responses to motivation or goal questions as factors explaining the probability of inclusion of alternative rock climbing sites in an independent availability model of choice set formation.

\section{Measurement and Estimation}

Whereas empirically oriented social scientists in the past had to make do with scarce databases or engage in costly primary data gathering, we live today in a golden age of relative data abundance. Due to the ubiquity of internet-enabled search (e.g., Google) and procurement (e.g., Amazon)—with matter-of-course recording of intermediate way stations and blind alleys - the ability to rigorously model not only eventual choice but the process of goal attainment has increased dramatically.

However, voluminous data is not synonymous with appropriate data, and many challenges remain for data collection, measurement, and inference when one wishes to estimate goal-based models of choice. The standard workhorse in the field is the discrete choice model, descending from McFadden's conditional logit and successively broadening to full-on Bayesian machinery to capture hierarchical choice processes, systematically and incidentally missing data, and data fusion [15]. But such methods are not designed to automatically model the intricate, time-bound processes of goal choice strategies that involve seeking, revising, and evaluating the attainment of goals (or lack thereof). Here, we consider some extensions, in terms of data requirements and model developments, towards that end specifically.

Let us consider again our examples in the domains of marketing, environmental public policy, transport, and the sociology of mate choice. Although these examples were chosen to highlight the vast range of goal choice strategies, they share certain features, some of them differentially implicated in goal orientation. Perhaps chief among these is the notion of constrained optimization: in the extant canonical choice theory setting, the decisionmaker must maximize outcome utility under a cost (time, money, cognition) constraint, but in a goal choice setting, options highly attractive in one goal's context may be ruled out by another. For example, in housing search, the "perfect home" may be struck for lacking a good neighborhood primary school, although one does not yet have children; a vacation consistent with the goal of "lifetime learning" may not score high on "fun with friends"; a family car that provides safety and suitable accoutrements may interfere with savings for children's college; or looking for an "established, stable provider" might translate into their already having had a family with a prior partner.

In each of these cases, the choices made by the decision-maker can involve not only the attributes and 
utility weights of others [3] but also the activation of, and conflict among, multiple goals, some of which are not traditionally part of the decision domain. For example, living in a part of the city that enables one to meet a wide variety of potential mates may entail rental costs that preclude purchasing a car or a home of one's own. As such, to calibrate not just models of choice but models of choice under multiple goal pursuit requires both a re-examination of constraint-based optimization and the sorts of data that that would require: how particular decisions not only impact goal attainment but also constrain seemingly unrelated options down the road for additional goals. Specific information that could inform a hierarchical setting would involve geo-demographics, lifestyle stage, financing and credit score information, as well as "market baskets" for both non-durable and durable products. Ideally, such information would be available for an entire co-habitating unit, to begin to unravel the multiple goals and constraints implicated by seemingly "individual" decisions.

To this end, data from a wide variety of choices and intermediate stages of choice would be especially useful: not only which widget was eventually purchased but also the full act of widget information search, across providers. To obtain anything like causal statements - e.g., "she did that because the goal of completing her education took precedence over purchasing a car"-researchers would ideally make use not only of field data but also of field experiments. For example, a web site offering information on homes for sale might differentially prioritize information pertinent to children (school quality, proximity to play areas, neighborhood composition, etc.) to determine how goals related to that affect site usage and eventual home choice. If such experiments are coordinated across providers - not only housing searches but also other information or purchase-oriented sites - a unified model of goals could be formulated from this sort of cross-context, individual-level data.

Even with such ideal data, the process of measurement is fraught with complexities. The sheer number of variables and their interactions would require either highly theory-driven, "top-down" searches for patterns (e.g., "we sought three types of consistent reaction") or what is essentially the opposite: a "bottom-up" machine-learning or nonparametric approach. So-called big data would not only enable but also practically invite the latter, where unsupervised learning could pick out salient patterns across data types while sidestepping attrition, missing information, and other distracting arcana of real data sets. Indeed, such a revolution has begun, with the automated extraction of marketing "meaning" from large corpora [57] and the use of high-dimensional nonparametric models to account for complex nonlinear and non-monotonic data relationships [8]. The use of such methods is presently constrained by storage, manipulation, and processing constraints, although recent advances in both classical and Bayesian estimation
[23], as well as the inexorable upward trajectory in computational speed, will increasingly enable researchers to fashion flexible models of goal attainment on available, large-scale data sources.

\subsection{Model Identification and a Partial Research Agenda for Testing the Model}

Combining Occam's razor and the need to search for evidence in outcome data (choices) brings us to the formulation of a key research question: When does a goal-based model of choice behavior provide better predictions of choice behavior than other (and simpler) models that do not feature a goal layer, and does the increase in predictive accuracy justify the loss in tractability?

The latter part of this question is essentially subjective, but the part preceding is objective in the sense that there are established procedures for determining the predictive performance of a choice model. A goal-based choice model's potential to generate better predictions than other models crucially depends on its ability to make predictions that differ from those made by other models (in the sense of assigning different choice probabilities to alternatives).

This also raises the question: what other models should one use as benchmark? Even though there are many ways to penalize model complexity in statistical testing procedures, comparing a full-fledged goal-based model with a linear-inparameters multinomial logit (MNL) model is not going to convince many scholars. For example, a choice model with a flexible error structure and a non-linear specification of observed utility may be a more credible candidate for goal-based models to be benchmarked against, rather than a simple MNL.

Combining these lines of argumentation, we suggest that the following research agenda be followed to arrive at a test of the added value of the goal-based framework proposed in this paper:

1. Find or collect data which in theory would allow the researcher to observe differences between the predictions made by goal-based models and competing choice models.

2. Specify the appropriate goal-based model, as well as a range of other candidate models that are likely to do well on the data; this class of competing models may include workhorse models such as linear-in-parameter MNL models, but should also include several more flexible and sophisticated models.

3. Estimate all models on the collected data and perform extensive out-of-sample validation tests. These validation tests can involve prediction of choices, but should also examine the ability of competing models to predict goals that subjects explicitly articulate (but are not used in estimation, of course). The objective of these tests should, at 
one level, be about establishing predictive validity, but this does not preclude examining the external and construct validity of models. Such tests are especially important when the goal-based model consumes more degrees of freedom (parameters) than competitor models.

4. Carefully inspect the differences in predictions between different models, if necessary at the level of individual choice situations/tasks. This may warrant the specific design of validation data collection efforts, separate from model estimation data. Explore if the goal-based model(s) differ from other models - in terms of predicted choice probabilities for particular alternatives - in ways that align well with the behavioral notions on which the model is built.

If the choice modeler's aim is simply to predict choice behavior, then the abovementioned steps - preferably performed on a large number of data sets by different teams of researchers - should suffice as a test of the potential of the goal-based approach. If one, in addition, has the ambition to derive managerial or policy implications, then a next step is to explore to what extent the goal-based approach may be translated into managerial or policy actions that differ from those generated by competitor models. And, ultimately, the desire is to construct a better understanding of the behavioral underpinnings of choice along with predictive ability.

\section{Discussion}

Currently popular models of choice behavior are econometrically quite sophisticated compared to the workhorse linear-in-parameters MNL models of the 1970s. Driven by scholars' ambitions to explain ever more subtle (latent) behavioral phenomena and aided by phenomenal advances in computational power, the choice modeler's toolbox has over the years been enriched with a wide variety of alternative model specifications. Initially, developments focused on incorporating more sophisticated error term structures to more closely represent observed substitution patterns, but more recently, doubtlessly inspired by the growing exploration of the judgment and decision theory literature and the increased popularity of behavioral economics, attention has shifted towards incorporating psychological insights in choice models.

In many instances, choice modelers with an interest in the behavioral sciences point at the often compelling intuitions underlying "behaviorally realistic" choice models. However, these intuitions (e.g., that people use higher-level goals when trading off attributes of choice alternatives) usually refer to the process of decision-making, while the data used to validate models tends to consist of decision outcomes (i.e., choices).
What is more, process data, such as verbal reports on mental processes or activated neurons in the case of fMRI studies, have been criticized for being unreliable.

This may require a Milton Friedman style "as if" conceptualization: since we are unable to rigorously verify the process assumptions underlying our model (i.e., the role of highlevel goals), we are forced to search for proof in observed choices. This challenge is not dissimilar to the early discussion in economics of the movement from demand for "goods" versus demand for attributes. Before Lancaster [29], there was not much discussion on the topic of attributes (at least in economics). It took a significant amount of time, and a different approach to data collection and analysis, before attribute-based approaches were fully employed in the literature. Another example of such shifts in approach is reference dependence: structural models embodying the concept have only appeared fairly recently, even though reduced form models that included reference dependence clearly worked better at predicting individual choice. Goals may present difficulties because they are generally unobservable, but that is also the case with reference dependence. The observer does not really know what people deem the reference condition to be, and it may not be the current state (the usual assumption made in analysis).

In Fig. 1, the double-headed arrow between the goal activation block and the goal-space representation is meant to convey the idea that the inability to attain one or more goals at a satisfactory level can lead to goal importance adaptation as well as (de)activation of other goals [33, 52]. Another way to state this is that people adapt to the decision context in part by changing their goal pursuit behavior if their desired goals are not attainable, perhaps to the point of deactivating some goals and activating others.

Readers will have noticed there has been little mention of the income or budget constraint (though see discussion following expression (2)). The traditional place to incorporate the income constraint is in attribute space, much in the form shown for constraint $H_{1}$ (see Fig. 1 again). From a process perspective, however, it seems to us that an alternative representation is that the budget and other exogenous constraints can be conceptualized following the selection of the preferred alternative(s) in goal space by checking for the budgetary impact of the choice(s) determined through goal pursuit. If the budget constraint is met, the decision-maker can move to implementation; however, if the budget constraint is violated by the desired outcome, it is necessary to return to earlier stages and implement constraints in attribute space or revise goal activations and importance so as to determine a final, feasible choice. An alternative depiction is that such constraints may be at work in the goal (de)activation stage (reduction of $\Gamma$ to $\Gamma_{\mathrm{A}}$ ), dictating which goals should be pursued and what importance should be assigned to them. Interesting possibilities arise from this second conceptualization: e.g., if 
someone projects that a budget constraint cannot be met for one goal if another is achieved (I may not be able to afford a car if I take a year off to travel to learn about shamanic traditions along the Silk Road, or I may not be able to support my family if I pursue my dream of being an artist), they may not activate certain goals. And as mentioned above, budget information may enter in the construction of process-based goals. The important issues here center on the stochasticity and future orientation of the activated goals under the influence of budget constraints.

\section{Conclusion}

In this paper, we have outlined key components of multiple, simultaneous goal pursuit and multi-stage choice, and have proposed how individuals' use of multiple goals can be incorporated in econometric models of individual decision-making. Since different goals are often only partially compatible, a multiple goal-based approach implies balancing goal leading to a deliberate goal-level choice strategy on the part of the decision-maker. Therefore, we introduced a conceptual framework that classified different aspects of individuals' decisions in the presence of multiple goals (Fig. 1). At the core of the proposed framework is the distinction between decision-makers' goal choice strategy (i.e., the individual's choice of establishing the role of goals, singly and in interaction; see [35]) and their goal evaluation strategy (i.e., a function of some or all of the attributes that affect the achievement of alternatives on a goal). The goal choice strategy is of prime importance in the proposed framework because it reflects the fact that the decision-maker's choice may ultimately be determined in goal space, not attribute space, as is more commonly represented. In that case, the goal evaluation strategy does not affect the choice directly but determines how well alternatives perform on each of the different goals. Thus, by discussing the main components as well as identification and modeling needs for the proposed goal-based choice strategies, we hope the current paper can stimulate further empirical research on the theoretically promising as well as practically relevant topic of individuals' choices in the presence of multiple goals.

Acknowledgments Benedict Dellaert and Bas Donkers thank Netspar for financial support for part of their research. The participation of Joffre Swait in this workshop was supported by the Australian Research Council Discovery Project DP140103966. Caspar Chorus would like to acknowledge support from the Netherlands Organization for Scientific Research (NWO Vidi-project 016-125-305).

Contribution Statement The aim of this paper is to provide directions on how individuals' use of multiple goals can be incorporated in econometric models of individual decision-making. Integrating goals in econometric models of choice allows for testing theories about goals on observed choice behavior and can lead to an improved understanding and prediction of individuals' choices. To achieve this objective, the paper combines findings from research on individuals' simultaneous pursuit of multiple goals with current advances in choice modeling.

Open Access This article is distributed under the terms of the Creative Commons Attribution 4.0 International License (http:// creativecommons.org/licenses/by/4.0/), which permits unrestricted use, distribution, and reproduction in any medium, provided you give appropriate credit to the original author(s) and the source, provide a link to the Creative Commons license, and indicate if changes were made.

\section{References}

1. Adamowicz WL, Swait JD (2013) Are food choices really habitual? Integrating habits, variety-seeking, and compensatory choice in a utility-maximizing framework. Am J Agric Econ 95:17-41

2. Arentze TA, Dellaert BG, Chorus CG (2015) Incorporating mental representations in discrete choice models of travel behavior: modeling approach and empirical application. Transp Sci 49(3):577-590

3. Aribarg A, Arora N, Kang MY (2010) Predicting joint choice using individual data. Mark Sci 29(1):139-157

4. Austin JT, Vancouver JB (1996) Goal constructs in psychology: structure, process, and content. Psychol Bull 120(3):338-375

5. Blamey RK (1996) Citizens, consumers and contingent valuation: clarification and the expression of citizen values and issue-opinions. In: Adamowicz WL, Boxall P, Luckert MK, Phillips WE, White WA (eds) Forestry, economics and the environment. CAB International, Wallingford, pp 103-133

6. Ben-Akiva M, McFadden D, Train K, Walker J, Bhat C, Bierlaire M, Bolduc D, Boersch-Supan A, Brownstone D, Bunch DS, De Palma A, Gopinath D, Karlstrom A, Munizaga MA (2002) Hybrid choice models: progress and challenges. Mark Lett 13(3):163-175

7. Bond SD, Carlson KA, Keeney RL (2010) Improving the generation of decision objectives. Decis Anal 7:238-255

8. Bruch E, Feinberg F and Lee KY (2016) Extracting multistage screening rules from online dating activity data. Proceedings of the National Academy of Sciences, 201522494

9. Chorus CG, Arentze TA, Timmermans HJ (2008) A random regretminimization model of travel choice. Transp Res B Methodol 42(1):1-18

10. Cooper RP, Ruh N, Mareschal D (2014) The goal circuit model: a hierarchical multi-route model of the acquisition and control of routine sequential action in humans. Cogn Sci 38:244-274

11. Dellaert BGC, Arentze TA, Timmermans HJ (2008) Shopping context and consumers' mental representation of complex shopping trip decision problems. J Retail 84(2):219-232

12. Dellaert BGC, Donkers B, Soest AV (2012) Complexity effects in choice experiment-based models. J Mark Res 49(3):424-434

13. Ding M (2007) A theory of intraperson games. J Mark 71(2):1-11

14. Etkin J (2016) Choosing variety for joint consumption in committed relationships. Journal of Marketing Research, forthcoming

15. Feit EM, Wang P, Bradlow ET, Fader PS (2013) Fusing aggregate and disaggregate data with an application to multiplatform media consumption. J Mark Res 50(3):348-364

16. Fiebig DG, Keane MP, Louviere J, Wasi N (2010) The generalized multinomial logit model: accounting for scale and coefficient heterogeneity. Mark Sci 29(3):393-421

17. Fishbach A, Ferguson MF (2007) The goal construct in social psychology. In: Kruglanski AW, Higgins TE (eds) Social psychology: handbook of basic principles. Guilford, NY, pp 490-515

18. FitzGerald THB, Dolan RJ, Friston KJ (2014) Model averaging, optimal inference, and habit formation. Front Hum Neurosci 8:457 
19. Gilbride TJ, Allenby GM (2004) A choice model with conjunctive, disjunctive, and compensatory screening rules. Mark Sci 23(3): 391-406

20. Glimcher PW and Fehr E (2014) Neuroeconomics: decision making and the brain. 2nd Edition. Elsevier

21. Gutman J (1982) A means-end chain model based on consumer categorization processes. J Mark 46(2):60-72

22. Hauser JR, Toubia O, Evgeniou T, Befurt R, Dzyabura D (2010) Disjunctions of conjunctions, cognitive simplicity, and consideration sets. J Mark Res 47(3):485-496

23. Hoffman MD, Gelman A (2014) The No-U-turn sampler: adaptively setting path lengths in Hamiltonian Monte Carlo. J Mach Learn Res 15(1):1593-1623

24. Kasser T (2016) Materialistic values and goals. Annu Rev Psychol 67:489-514

25. Keeney RL (2009) The foundations of collaborative group decisions. International Journal Collaborative Engineering, 1 1(1-2): 4-18

26. Kim DS, Bailey RA, Hardt N and Allenby GM (2016) Benefitbased conjoint analysis. Marketing Science, forthcoming

27. Konovalov A, Krajbich I (2016) Over a decade of neuroeconomics: what have we learned? Organ Res Methods. doi:10.1177/ 1094428116644502

28. Kopetz CE, Kruglanski AW, Arens ZG, Etkin J, Johnson HM (2012) The dynamics of consumer behavior: a goal systemic perspective. J Consum Psychol 22(2):208-223

29. Lancaster KJ (1966) A new approach to consumer theory. J Polit Econ 74(2):132-157

30. Larrick RP (2016) The social context of decisions. The Annual Review of Organizational Psychology and Organizational Behavior. doi:10.1146/annurevorgpsych-041015-062445

31. Levitt S, List JA (2007a) Viewpoint: on the generalizability of lab behaviour to the field. Can J Econ 40(2):347-370

32. Levitt S, List JA (2007b) What do laboratory experiments measuring social preferences reveal about the real world. J Econ Perspect 21(2):153-174

33. Li L (2013) The role of antecedent volition on consumer evaluative processes and choice behavior, Doctoral Dissertation, School of Business, University of Alberta, Fall 2013

34. Luce MF, Payne JW, Bettman JR (2000) Coping with unfavorable attribute values in choice. Organ Behav Hum Decis Process 81(2): 274-299

35. Marley AAJ, Swait J (2017) Goal-based models for discrete choice analysis. Transp Res B 101:72-88

36. McFadden D (1980) Econometric models for probabilistic choice among products. Journal of Business, S13-S29

37. McFadden D, Train K (2000) Mixed MNL models for discrete response. J Appl Econ 15:447-470

38. Morey E, Thiene M (2012) A parsimonious, stacked latent-class methodology for predicting behavioral heterogeneity in terms of life-constraint heterogeneity. Ecol Econ 74:130-144

39. Myers JH (1976) Benefit structure analysis: a new tool for product planning. J Mark 40(4):23-32

40. O'Reilly RC, Hazy TE, Mollick J, Mackie P, and Herd S (2014) Goal-driven cognition in the brain: a computational framework. Manuscript, Department of Psychology and Neuroscience, University of Colorado Boulder
41. Olson DL, Mechitov AI and Moshkovich H (1999) Comparison of MCDA paradigms. In N. Meskens and M. Rouben (eds.) Advances in decision modelling, pp. 105-119

42. Oppenheimer VK (1988) A theory of marriage timing. Am J Sociol 94:563-591

43. Orehek E, Forest AL (2016) When people serve as means to goals: implications of a motivational account of close relationships. Curr Dir Psychol Sci 25:79-84. doi:10.1177/0963721415623536

44. Paulssen M, Temme D, Vij A, Walker J (2014) Values, attitudes and travel behavior: a hierarchical latent variable mixed logit model of travel mode choice. Transportation 41(4):873-888

45. Romero C (2004) A general structure of achievement function for a goal programming model. Eur J Oper Res 153:675-686

46. Saaty TL (2008) Decision making with the analytic hierarchy process. International Journal of Services Sciences 1:83-98

47. Savage LJ (1954) The foundations of statistics. John Wiley \& Sons and Chapman \& Hall, New York

48. Souza F (2015) Goal-based choice set formation, Doctoral Dissertation, Institute for Choice, Business School, University of South Australia, March, 146pp

49. Steg L, Perlaviciute G, van der Werff E, Lurvink J (2014) The significance of hedonic values for environmentally relevant attitudes, preferences, and actions. Environ Behav 46(2):163-192

50. Swait J (2001) A non-compensatory choice model incorporating attribute cutoffs. Transportation Research Part B: methodological 35(10):903-928

51. Swait J, Adamowicz W (2001) The influence of task complexity on consumer choice: a latent class model of decision strategy switching. J Consum Res 28(1):135-148

52. Swait J, Li L, Argo J (2017) Modeling simultaneous multiple goal pursuit and adaptation in consumer choice. Working Paper. Institute for Choice, University of South Australia, Sydney

53. Swait J, Marley AAJ (2013) Probabilistic choice (models) as a result of balancing multiple goals. J Math Psychol 57:1-14

54. Taatgen NA, Huss D, Dickison D, Anderson JR (2008) The acquisition of robust and flexible cognitive skills. J Exp Psychol Gen 137:548-565

55. Thiene M, Swait J, Scarpa R (2017) Choice set formation for outdoor destinations: the role of motivations and preference discrimination in site selection for the management of public expenditures on protected areas. J Environ Econ Manag 81:152-173

56. Ter Hofstede F, Steenkamp JBE, Wedel M (1999) International market segmentation based on consumer-product relations. J Mark Res 36(1):1-17

57. Tirunillai S, Tellis GJ (2014) Mining marketing meaning from online chatter: strategic brand analysis of big data using latent dirichlet allocation. J Mark Res 51(4):463-479

58. Van Osselaer SM, Janiszewski C (2012) A goal-based model of product evaluation and choice. J Consum Res 39(2):260-292

59. Von Neumann, J. and Morgenstern, O. (1947). Theory of games and economic behavior. 2nd rev. ed, Princeton: Princeton University Press.

60. Weber EU, Johnson EJ (2009) Mindful judgment and decision making. Annu Rev Psychol 60:53

61. Wood W, Runger D (2016) Psychology of habit. Annu Rev Psychol 67:289-314. doi:10.1146/annurev-psych-122414-033417 